\title{
Necessity of Reticulocyte Calibration for More Accurate and Precise Results
}

\author{
Yong Kwan Lim, M.D. ${ }^{1}$, Hyun-Young Chi, M.D. ${ }^{2}$, Mi-Kyung Lee, M.D. ${ }^{1}$, and Hye Ryoun Kim, M.D. ${ }^{1}$ \\ Department of Laboratory Medicine ${ }^{1}$, Chung-Ang University College of Medicine, Seoul; Samkwang Medical Laboratories², Seoul, Korea
}

\section{Dear Editor,}

Reticulocyte count and immature reticulocyte fraction (IRF) are known to be accurate indices of erythropoietic activity [1]. Apart from clinical utility, these parameters are also useful for detecting blood doping in the athletic field [2, 3]. Recently, the XN system (Sysmex, Kobe, Japan) was introduced to the market [4]. Although this new instrument uses the same analytical method for reticulocyte counting as the previous version of the XE system, the number of red blood cells (RBCs) that could be counted during reticulocyte counting was increased to improve the precision; in addition, the XN system introduced a new calibrator (XN CAL, Sysmex) to harmonize the reticulocyte count among different analyzers. Increase in the number of RBCs that can be counted and calibration of the reticulocyte count could contribute to the interchangeability of the results, not only among hospitals but also among anti-doping laboratories. The aim of this study was to demonstrate and quantify the improvement in the performance of reticulocyte parameters in the XN series with calibration.

For comparative analysis, $40 \mathrm{~K}_{2}$ EDTA-treated samples were used to compare the results from automated hematology analyzers (XN-9000 and XE-2100) with that from the reference method for reticulocyte counting (new methylene blue staining procedure) $[5,6]$. All whole-blood samples were the remnants of patient samples that had been requested for a routine com- plete blood cell count in Chung-Ang University Hospital, Seoul, Korea. The study was conducted in Chung-Ang University Hospital between April and June 2016, and ethical approval was granted by the Chung-Ang University Hospital ethics committee (C2016053[1796]).

Precision analysis was carried out on three levels of quality control materials (XN CHECK ${ }^{\mathrm{TM}}$, Sysmex). Based on the CLSI document EP05-A3 [7], each sample was tested 80 times, with two replicate measurements per run and two runs per day for 20 days. The results of the comparative study were analyzed using Deming regression analysis in SigmaPlot 12.0 (Systat Software Inc., CA, USA). The repeatability and within-laboratory precision were calculated with 95\% confidence intervals using CLSI EP05-A3 [7] in Excel 2010 (Microsoft, WA, USA).

Comparative analysis of reticulocyte counts revealed excellent correlation between the reference method and XN-9000 and XE-2100 (Fig. 1). Results from XN-9000 perfectly matched those from the reference method (slope $=0.9883$, intercept $=0.0142$ ), but the results of the reticulocyte count from XE-2100 were consistently lower than those from the reference method (slope $=$ 0.9110 , intercept $=-0.0680$ ). The IRF results showed high correlation, but XN-9000 yielded consistently higher results than XE-2100 (Fig. 2). The discrepancy in the paired results was pronounced when the IRF values were especially high.
Received: July 15, 2017

Revision received: August 7, 2017

Accepted: January 15, 2018

\section{Corresponding author: Hye Ryoun Kim}

Department of Laboratory Medicine, Chung-Ang University College of Medicine, 102 Heukseok-ro, Dongjak-gu, Seoul 06910, Korea

Tel: +82-2-6299-2718, Fax: +82-2-6298-8630, E-mail: hyekim@cau.ac.kr

\section{(C) Korean Society for Laboratory Medicine}

This is an Open Access article distributed under the terms of the Creative Commons Attribution Non-Commercial License (http://creativecommons.org/licenses/by-nc/4.0) which permits unrestricted non-commercial use, distribution, and reproduction in any medium, provided the original work is properly cited. 
A

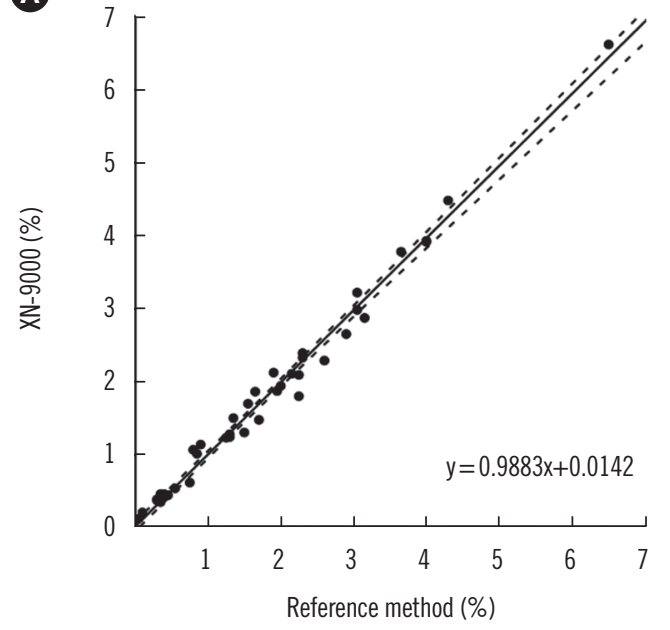

C

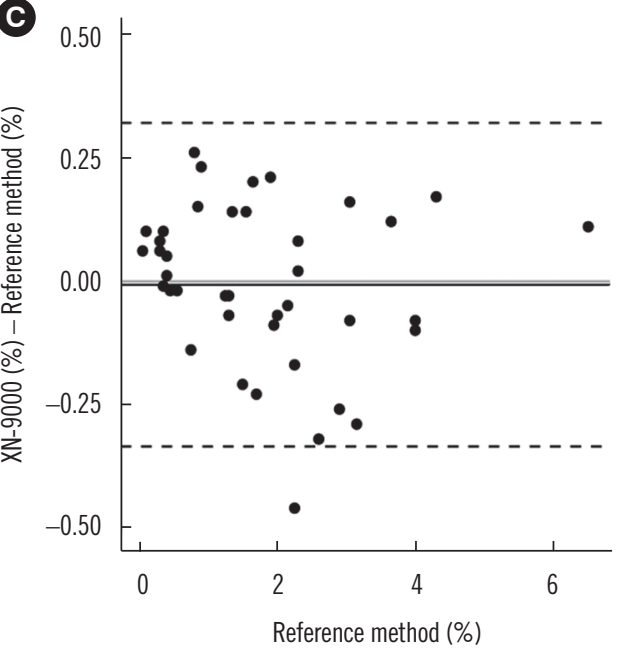

B

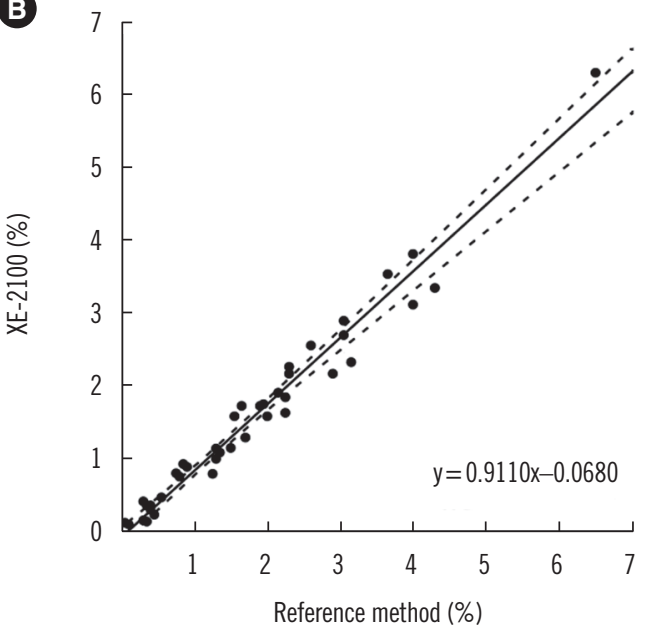

(D)



Fig. 1. Correlation graphs with $95 \%$ confidence intevals (A, B) and difference plots (C, D) of reticulocyte counts from analyzers XN-9000 and XE-2100 against the reference method. In the difference plots, the bold lines and dashed lines represent bias and the $95 \%$ limits of agreement, respectively.

The repeatability for the reticulocyte counts and absolute reticulocyte counts ranged from $2.31 \%$ to $3.55 \%$ for XN-9000 and from $3.36 \%$ to $4.31 \%$ for XE-2100. The within-laboratory precision values of the reticulocyte count and absolute reticulocyte count were between $2.79 \%$ and $3.83 \%$, respectively, for XN-9000 and between 3.24\% and 4.65\%, respectively, for XE2100 . As for IRF, the repeatability values ranged from $5.28 \%$ to $7.04 \%$ for XN-9000 and from $13.71 \%$ to $15.54 \%$ for XE-2100, and the within-laboratory precision values ranged from $8.58 \%$ to $10.40 \%$ for XN-9000 and from $14.97 \%$ to $16.28 \%$ for XE2100. Compared with XE-2100, XN-9000 showed improved precision for all the parameters.

Our study showed that the reticulocyte count estimated using $\mathrm{XN}-9000$ was almost identical to that estimated using the refer- ence method. In contrast, XE-2100 always underestimated the reticulocyte count and the results were consistently approximately $9 \%$ lower than the results of the reference method (mean bias= $0.23 \%$ ). This difference should be calibrated against the reference method because the measured results are clearly very different from the actual values. When we compared the precision of reticulocyte counts, XN-9000 showed significantly improved precision with all measured parameters (reticulocyte count and IRF) at all concentrations. We hypothesized that this improvement was caused by the increase in RBC count during reticulocyte counting, and calibrating the RBC count may improve the precision of the reticulocyte count, as this precision depends on the stability of the instrument response [8].

For IRF measurement, there were significant differences be- 


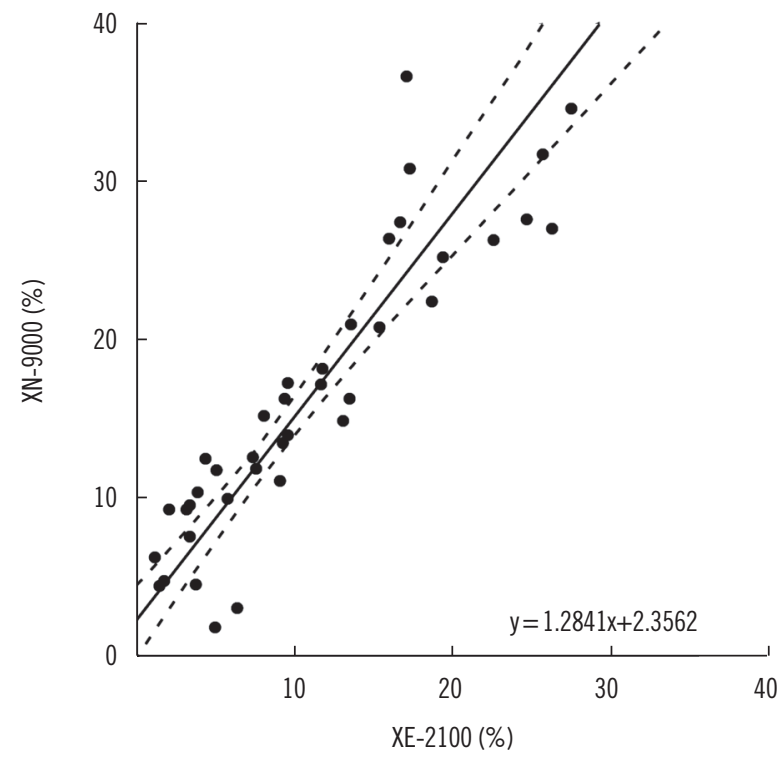

Fig. 2. Correlation graph of the immature-reticulocyte fraction between analyzers XN-9000 and XE-2100. The dashed lines represent the $95 \%$ confidence intevals.

tween the two analyzers. Because of the lack of a reference method, the results of IRF depend on methodological differences such as differences in fluorescent dyes and high-fluorescence reticulocytes thresholds [9]; however, our data revealed that IRF results were not interchangeable between the XN and XE systems, even though the manufacturer, method, and principle were identical.

In summary, the calibration of the reticulocyte count has immensely improved the overall performance of methods for measuring the reticulocyte parameters. For accurate measurement of reticulocytes, we recommend the $\mathrm{XN}$ system as a reliable hematological analyzer. For IRF measurement, there are evident differences even among hematological analyzers made by the same manufacturer. Therefore, there is a need for a reference method and harmonization of IRF measurements to minimize instrument-to-instrument variation.

\section{Authors' Disclosures of Potential Conflicts of Interest}

There are no conflicts of interest to declare.

\section{Acknowledgment}

This research was supported by the Basic Science Research Program through the National Research Foundation of Korea (NRF), funded by the Ministry of Education (NRF-2017R1A2B4011631). The authors would like to thank the department of hematology of the Chung-Ang University Hospital for their contribution to this study.

\section{REFERENCES}

1. Chang CC and Kass L. Clinical significance of immature reticulocyte fraction determined by automated reticulocyte counting. Am J Clin Pathol 1997; 108:69-73.

2. WADA, Blood analytical requirements for the athlete biological passport. In: World anti-doping agency. Athlete biological passport operating guidelines and compilation of required elements. V5.0. World Anti-Doping Agency, 2014:31-4.

3. Sottas PE, Robinson N, Giraud S, Taroni F, Kamber M, Mangin P, et al. Statistical classification of abnormal blood profiles in athletes. Int J Biostat 2006;2:1-21.

4. Briggs C, Longair I, Kumar P, Singh D, Machin SJ. Performance evaluation of the Sysmex haematology XN modular system. J Clin Pathol 2012; 65:1024-30.

5. Briggs C, Culp N, Davis B, d'Onofrio G, Zini G, Machin SJ. ICSH guidelines for the evaluation of blood cell analysers including those used for differential leucocyte and reticulocyte counting. Int J Lab Hematol 2014; 36:613-27.

6. CLSI. Methods for Reticulocyte Counting (Automated Blood Cell Counters, Flow Cytometry, and Supravital Dyes). 2nd ed. CLSI supplement EP44-A2. Wayne, PA: Clinical and Laboratory Standards Institute; 2004.

7. CLSI. Evaluation of Precision of Quantitative Measurement Procedures. 3rd ed. CLSI supplement EP05-A3. Wayne, PA: Clinical and Laboratory Standards Institute; 2014.

8. Burtis CA, Ashwood ER, Bruns DE, Tietz NW. Tietz textbook of clinical chemistry and molecular diagnostics. 5th ed. St. Louis, Mo.: Saunders, 2013.

9. Briggs C, Grant D, Machin SJ. Comparison of the automated reticulocyte counts and immature reticulocyte fraction measurements obtained with the ABX Pentra 120 Retic blood analyzer and the Sysmex XE-2100 automated hematology analyzer. Lab Hematol 2001;7:75-80. 